\title{
Dr. Gisela Niemeyer, Richterin des Bundesverfassungsgerichts a.D. Zur Erinnerung
}

Prof. Dr. Helga Oberloskamp, Bonn

Am 7. Februar 2012 ist die ehemalige Richterin des Bundesverfassungsgerichts Frau Dr. Gisela Niemeyer im Alter von 88 Jahren in Bonn verstorben. Gisela Niemeyer war 33 Jahre lang Mitglied des djb und hat sich besonders in den Zeiten des politischen Bonn in der dortigen Ortsgruppe engagiert. Ich selbst habe sie darüber hinaus als Finanzrichterin des FG Köln, bei dem ich anstelle des Verwaltungsgerichts eine Station absolviert habe, und als Bundesverfassungsrichterin bei Besuchen mit meinen Studenten näher kennenlernen dürfen.

Frau Niemeyer ist in Danzig geboren, wo sie auch ihre Schulzeit verbrachte, die sie 1942 mit dem Abitur beendete. Ihren Wunsch, Jura zu studieren, konnte sie erst 1948 verwirklichen, weil sie sich zuvor mit Reichsarbeitsdienst und Kriegshilfsdienst (als Aushilfslehrerin), einem als Vorwand gewählten Medizinstudium (bis zum Vorphysikum) und schließlich erneutem Kriegshilfsdienst (in einem Wehrertüchtigungslager in Österreich) bewähren musste. Nach der Ausweisung von Danzig nach Hameln/ Weser arbeitete sie zunächst als Verwaltungsangestellte.

Zum Sommersemester 1948 endlich konnte sie beruflich das machen, was sie von Anfang an geplant hatte. Nach sechs Semestern Jurastudium in Kiel (1951 Heirat und Führen einer Studentenehe mit Kind) absolvierte sie ihr Erstes Juristisches Staatsexamen. Danach musste sie jedoch ihren weiteren juristischen Werdegang erneut unterbrechen und zunächst als Verwaltungsangestellte im Justizministerium in Bonn ihren Lebensunterhalt verdienen. Von 1953 bis 1956 absolvierte sie schließlich ihren juristischen Vorbereitungsdienst und schloss ihn mit dem Zweiten Juristischen Staatsexamen am Oberlandesgericht Köln ab. Ihre akademische Karriere beendete sie 1961 mit ihrer Promotion zum Thema „Der Gegenstand des Verfahrens bei der Anfechtung von Steuerbescheiden“.

Schon zuvor hatte Frau Niemeyer ihre praktische juristische Laufbahn als Finanzassessorin, Regierungsassessorin,
Regierungsrätin sowie Oberregierungsrätin an verschiedenen Orten in Nordrhein-Westfalen (Sachgebietsleiterin beim Finanzamt Bonn-Innenstadt, Lehrerin und Leiterin der Landesfinanzschule Nordkirchen) begonnen. 1966 wurde sie Finanzgerichtsrätin und sodann Senatspräsidentin am Finanzgericht Düsseldorf. Von 1972 bis 1975 war sie Bundesrichterin beim Bundesfinanzhof und dort die erste Frau seit Bestehen des Reichs- und Bundesfinanzhofs. Sie beschloss ihre finanzgerichtliche Karriere mit ihrer Ernennung zur Präsidentin des Finanzgerichts Düsseldorf, womit sie ebenfalls die erste Präsidentin eines Finanzgerichts in der Bundesrepublik war.

Im November 1977 wurde Frau Niemeyer zur Richterin des Ersten Senats des Bundesverfassungsgerichts ernannt, wo sie die dritte Frau (Nachfolgerin von Dr. h.c. Wiltraut Rupp-von Brünneck) seit Bestehen des Gerichts war. Dr. Erna Scheffler, die erste Frau, bezeichnete sie als „ihr Enkelkind“. Zehn Jahre lang blieb sie die einzige Frau am Bundesverfassungsgericht überhaupt. In ihrer Eigenschaft als Bundesrichterin war sie Berichterstatterin für das 1. Eherechtsreformgesetz (besonders bedeutsam der Versorgungsausgleich). Bleibende juristische Spuren hat sie bis heute mit ihrer Rechtsprechung zum Pflegekind hinterlassen. 1989 ging sie nach einem wahrlich nicht abwechslungsarmen Berufsleben in ihren wohlverdienten Ruhestand. Für ihre besonderen Verdienste wurde sie mit dem großen Verdienstkreuz mit Stern und Schulterband des Verdienstordens der Bundesrepublik Deutschland ausgezeichnet.

Die letzten Jahre ihres Lebens verbrachte Frau Niemeyer mit ihrem Ehemann Günther Niemeyer in einer Seniorenresidenz in Bonn-Bad Godesberg. Ihre ebenfalls im Bonner Raum lebende Tochter kümmerte sich um die Eltern.

Mit Frau Niemeyer hat der djb ein fachlich großartiges Mitglied und eine herausragende Persönlichkeit unter den Juristinnen der Bundesrepublik Deutschland verloren. 